\title{
Models based on variable fractional digestion rates to describe ruminal in situ digestion*
}

\author{
BY JAAP VAN MILGEN† AND RENÉ BAUMONT \\ Institut National de la Recherche Agronomique, Station de Recherche sur la Nutrition des \\ Herbivores, Centre de Clermont-Ferrand Theix, 63122 Saint Genès-Champanelle, France
}

(Received 25 October 1993 - Revised 12 September 1994-Accepted 30 September 1994)

\begin{abstract}
Using a first-order model to describe ruminal in situ digestion implies that the rate of digestion is affected only by the quantity of potentially digestible substrate remaining. Other factors, like the microbial efficacy for digesting substrate, are assumed to be constant. However, microbes are not only the cause but also the result of digestion, being one of the digestion end-products. Two sigmoidal models (a logistic and a Gompertz-like model) were derived from a general digestion function in which the rate of digestion equals the product of the quantity of potentially digestible substrate remaining and a non-constant fractional rate of digestion (microbial efficacy function). The models were compared with a first-order model with a discrete lag time. The logistic model specifically accounted for the conversion of substrate mass to microbial mass, but did not describe microbial migration between the substrate and the ruminal fluid. In contrast, the Gompertz-like model assumed that the change in microbial efficacy was only timedependent. There was little difference between models in estimates of scale parameters, but the asymptotic microbial efficacy was consistently higher for the logistic model than for the other models. Estimates of discrete lag time in the first-order model were biased towards obtaining values identical to the independent variable. Scale estimators appeared to be more robust than kinetic estimators. Lack-offit was present for most model-data set combinations. The similar patterus of residuals between models suggested that a four-parameter model may be insufficient to describe the data. It was concluded that if a four-parameter model is to be used, the model with a discrete lag time would be the least biologically appropriate.
\end{abstract}

Models: Rumen digestion: Microbial activity

In vivo and in situ incubation of feeds serves as a basic procedure in many feed evaluation systems. The formulation of hypotheses concerning underlying biological concepts has led to the development of different mathematical models describing digestion in the rumen. The use of models allows comparison of (functions of) parameter estimates, which ideally reflect these biological concepts, between feeds or feeding systems.

Digestion of particulate matter in the rumen is a microbial enzymic process but is generally described by first-order kinetics with a constant fractional (or specific) rate of digestion:

$$
\frac{1}{S} \frac{\mathrm{d} S}{\mathrm{~d} t}=-k
$$

where $S$ is the quantity of potentially digestible substrate remaining $(\mathrm{g} / \mathrm{g}$ incubated), and

* Presented in part at the 8th Conference on Nutrition and Feeding of Herbivores, Paris, 24-25 March 1993 (van Milgen, 1994).

$\dagger$ Current address: Institut National de la Recherche Agronomique, Station de Recherches Porcines, Centre de Recherches de Rennes, 35590 Saint-Gilles, France. 
$k$ is the fractional rate of digestion $(/ \mathrm{h})$. The rate of digestion (the absolute value of $\mathrm{d} S / \mathrm{d} t$ ) is proportional to the quantity of potentially digestible substrate and decreases continuously. Other factors affecting digestion (e.g. microbes, enzymes, surface area) are therefore assumed to be constant, their effects described by the fractional rate constant. However, it has been shown that the rate of digestion for many feedstuffs initially increases rather than decreases (Mertens, 1973; Sauvant et al. 1985). This has been explained by suggesting that not all potentially digestible substrate is available for digestion immediately upon incubation. This delayed availability of substrate has been represented by discrete (Mertens \& Loften, 1980; McDonald, 1981) and dynamic lag phases (van Milgen et al. 1991).

Digestion of particulate substrates is caused by ruminal microbes that attach to, or are in close proximity to the (primarily inner) particle surfaces. However, the number of attached microbes can change remarkably during incubation in the rumen (Cheng et al. 1980; van Milgen et al. 1993). As a result, the effect of microbes (and thus enzymes) on digestion of substrate may not be constant. This can be an alternative explanation for the increased rate of digestion during early incubation. The purpose of the present study was to identify models accommodating the assumption that the fractional rate of digestion is not constant, but varies with incubation time. The proposed models will differ from the classical model only in the way the dynamics of digestion are described; assumptions concerning the fractionation of substrate will remain unchanged. The models will be derived from a general digestion model and their biological, mathematical, and statistical properties will be discussed.

\section{MATERIALS AND METHODS}

Data

Data obtained from an in situ study published by van Milgen et al. (1993) were used to evaluate the models. In short, air-dry lucerne hay (Medicago sativa), maize cobs (Zea mays), orchard-grass hay (Dactylis glomerata), and wheat straw (Triticum aestivum) were ground to pass through a $4 \mathrm{~mm}$ screen and portions of approximately $4 \mathrm{~g}$ were weighed into multi-filament, dacron bags $(70 \times 130 \mathrm{~mm}$; pore size $50 \mu \mathrm{m})$. Sets of eight dacron bags (four substrates, two bags per time point for each substrate) were put into loose-mesh sacks, which were anchored with a weight. Sacks were incubated below the particulate mat layer in the rumen of a steer for $0 \cdot 5,1,1 \cdot 5,2,3,4,6,9,13,18,24,31,40,54,77$ and $154 \mathrm{~h}$. Eight sacks $(t 0.5$ to $t 9 \mathrm{~h}$ ) were incubated at 08.00 hours, whereas the remaining sacks ( $t 13$ to $t 154 \mathrm{~h}$ ) were incubated at 20.00 hours. Bags for which $t=0$ were not incubated in the rumen but underwent the same rinsing procedures as the other bags. The steer was fed twelve times daily with a mixed forage diet based on the four substrates. Details of diet composition, management, and analyses are given by van Milgen et al. (1993).

\section{Fractionation}

Substrate incubated in the rumen can be divided into three discrete fractions. The soluble fraction $\left(f_{s} ; \mathrm{g} / \mathrm{g}\right.$ incubated $)$ is often defined as material that is lost during the washing procedure before in situ fermentation. The insoluble substrate is taken to be composed of a potentially digestible fraction $\left(f_{d} ; \mathrm{g} / \mathrm{g}\right.$ incubated $)$ and an indigestible fraction $\left(f_{i} ; \mathrm{g} / \mathrm{g}\right.$ incubated). Because it is assumed that not all substrate is digestible in the ruminal environment, $f_{i}$ is estimated as the quantity of substrate remaining after infinite ruminal incubation. Models developed here are based on the assumption of homogeneity of potentially digestible substrate. 


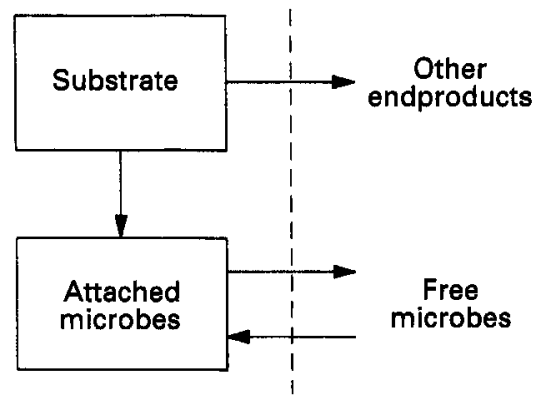

Fig. 1. General model describing in situ digestion of substrate. The boundary (dashed line) separates the system (substrate and attached microbes in the bag) from the environment (ruminal liquid).

\section{General digestion model}

Fig. 1 depicts a hypothetical model for in situ digestion of substrate. The model assumes the existence of two pools of microbes, a pool of microbes associated with the ruminal liquid (free microbes) and a pool of microbes associated with, or in close proximity to, the substrate (attached microbes). The pool of free microbes in the rumen is large and relatively constant compared with the pool of attached microbes in the bags.

Upon placement in the rumen, few microbes which can digest the substrate in the ruminal environment are present in and on the particles (Cheng et al. 1980). Microbes, present in rumen fluid, will enter particles via stomata and sites of epidermal lesions and their migration may be mediated through chemotaxis (Cheng et al. 1980; Chesson \& Forsberg, 1988). However, it is unlikely that all microbes that are present within a particle (or in situ bag) originate directly from the rumen fluid. Once attached, energy and nutrients resulting from digestion will be used for microbial maintenance and growth. Microbial growth (i.e. the increase in the number of microbes) therefore occurs in close proximity to the site of substrate digestion. If sufficient potentially digestible substrate is available, newly synthesized microbes are also likely to attach in close proximity to the site of digestion. Apparently, digestion of particulate matter is at least partly an autocatalytic process because microbes are not only the cause but also the result of digestion.

The proposed model, although a simplification of reality (e.g. it does not distinguish between maintenance and growth requirements of the microbes), is too complex to be used as a tool for analysis of in situ data. As indicated above, rate of digestion is limited initially by attachment of bacteria to the particle surfaces. Once the inner surfaces are extensively colonized, rate of digestion cannot be increased further by entry of microbes from the environment or synthesis of microbial mass within the particles. The effect of microbes on digestion of substrate, then, is constant and the rate of digestion is proportional to the quantity of substrate remaining (i.e. a first-order process). Models that will be presented here are based on the concept that the effect of microbes is limiting to digestion during early incubation, and constant after prolonged incubation. In a similar way to Michaelis-Menten kinetics, rate of disappearance of potentially digestible substrate from the system is described by a mass-action process, i.e.:

$$
\frac{\mathrm{d} S}{\mathrm{~d} t}=-k S M
$$

where $M$ is the quantity of microbial matter attached to, or in close proximity to, the substrate ( $\mathrm{g}$ microbes/g substrate incubated), and $k$ is a constant ((g microbes/g substrate 


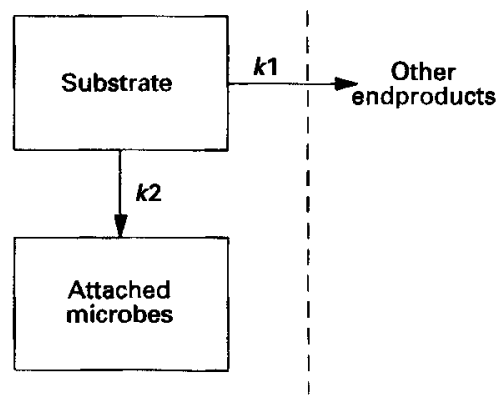

Fig. 2. Logistic model describing in situ digestion of substrate.

incubated)/h). Because the quantity of microbial matter is seldom measured during most in situ studies, the product of $k$ and $M$ can be replaced by a more general function $Z$ :

$$
\frac{\mathrm{d} S}{\mathrm{~d} t}=-S Z
$$

where $Z$ is a microbial efficacy function $(/ \mathrm{h})$ which represents the effect, rather than the quantity, of microbes (or enzymes) on substrate digestion. The quantity of substrate remaining in the bag $(Y)$ is then given by:

$$
Y=\int_{0}^{t}-S Z \mathrm{~d} t+f_{i}, \quad \text { with } S(0)=f_{d} .
$$

\section{Logistic model}

The logistic model (Fig. 2) was derived by assuming that there is no exchange of microbes between the system and the environment. If, as before, $S$ is the quantity of potentially digestible substrate and $M$ is the quantity of microbial matter, the system is described by the following differential equations:

$$
\begin{gathered}
\frac{\mathrm{d} S}{\mathrm{~d} t}=-\left(k_{1}+k_{\mathrm{2}}\right) S M, \\
\frac{\mathrm{d} M}{\mathrm{~d} t}=k_{2} S M .
\end{gathered}
$$

If digestion end-products (i.e. microbes and other end-products) are produced in constant proportion and $f_{d}$ is the initial value of $S$, then $\left(f_{d} k_{2}\right) /\left(k_{1}+k_{2}\right)$ will be converted quantitatively into microbes, and $\left(f_{d} k_{1}\right) /\left(k_{1}+k_{2}\right)$ to other digestion end-products. Because digestion would never commence with $M(0)=0$, a non-zero initial value for $M$ is required. The initial quantity of microbial matter plus the quantity of substrate that eventually will be converted into microbes is constant (i.e. $M_{\infty}=M_{0}+\left(f_{d} k_{2}\right) /\left(k_{1}+k_{2}\right)$, where $M_{\infty}$ is the quantity of microbial matter after infinite incubation). The $M_{\infty}$ is also equal to the current quantity of microbial matter and the current quantity of substrate that has not yet been converted quantitatively into microbial matter $\left(M_{\infty}=M+\left(S k_{2}\right) /\left(k_{1}+k_{2}\right)\right)$. Rearranging terms and substitution of $\left(S k_{2}\right)$ with $\left(M_{\infty}-M\right)\left(k_{1}+k_{2}\right)$ in equation (4) yields:

$$
\frac{\mathrm{d} M}{\mathrm{~d} t}=\left(k_{1}+k_{2}\right)\left(M_{\infty}-M\right) M \text {. }
$$

The microbial efficacy function $(Z)$ was defined to express the effect, rather than the quantity of microbial matter. This effect can be calculated from equations (1) and (3) (i.e. 
$\left.Z=\left(k_{1}+k_{2}\right) M\right)$. Likewise, $\left(k_{1}+k_{2}\right)\left(M_{\infty}-M\right)$ can be replaced by $\left(Z_{\infty}-Z\right)$. The function $Z$ has the same properties as $M$, only the scaling is different. Therefore, $(\mathrm{d} Z / \mathrm{d} t) / Z=$ $(\mathrm{d} M / \mathrm{d} t) / M$ and the rate of change of $Z$ is:

$$
\frac{\mathrm{d} Z}{\mathrm{~d} t}=\left(Z_{\infty}-Z\right) Z,
$$

which yields after integration with $Z_{0}$ (the initial value of $Z$, which also equals $\left.\left(k_{1}+k_{2}\right) M_{0}\right)$ :

$$
Z=\frac{Z_{\infty} Z_{0}}{Z_{0}+\left(Z_{\infty}-Z_{0}\right) \exp \left(-Z_{\infty} t\right)} .
$$

Substitution of $Z$ in equation (2) results after integration in:

$$
Y=\frac{f_{d} Z_{\infty}}{Z_{0} \exp \left(Z_{\infty} t\right)+Z_{\infty}-Z_{0}}+f_{i},
$$

where $Y$ is the mass of the substrate remaining in the in situ bags (g/g incubated), and $Z_{0}$ and $Z_{\infty}$ are the initial and asymptotic values of the microbial efficacy function, respectively $(/ \mathrm{h})$.

\section{Gompertz-like model}

An alternative hypothesis is to assume that the microbial efficacy function is a timedependent process, caused by both autocatalysis and exchange of microbes between the system and the environment. Although such a process would not explicitly account for either phenomenon, it can have properties that cannot be incorporated into the logistic model. As indicated earlier, digestion is limited initially by the availability of microbes on substrate surfaces. However, after prolonged incubation the number of microbes might exceed the number of attachment sites. When the surfaces of particles are extensively colonized, rate of digestion cannot be increased further by the presence of additional microbes. Therefore, the microbial efficacy function $(Z)$ should intersect the origin, and have an asymptotic value after prolonged incubation. Equation (6) represents this concept:

$$
\frac{\mathrm{d} Z}{\mathrm{~d} t}=\left(Z_{\infty}-Z\right) k_{z},
$$

where $Z_{\infty}$ is the asymptotic value of $Z(/ \mathrm{h})$, and $k_{z}$ the rate constant for the change of $Z$ (/h). Integration of equation (6) with $Z(0)=0$ yields:

$$
Z=Z_{\infty}\left(1-\exp \left(-k_{z} t\right)\right) \text {. }
$$

Substitution of $Z$ in equation (2) results after integration in an equation that can be used for analysis of in situ data:

$$
Y=f_{d} \exp \left(\frac{Z_{\infty}\left(1-k_{z} t-\exp \left(-k_{z} t\right)\right)}{k_{z}}\right)+f_{i} .
$$

Model with discrete lag time

The logistic and Gompertz-like models were compared with a first-order model with a discrete lag time (Mertens \& Loften, 1980; McDonald, 1981). This model assumes that $Z=0$ before lag time $\left(t_{\text {lag }}, \mathrm{h}\right)$ and that $Z=Z_{\infty}$ thereafter:

$$
\begin{aligned}
& Y=f_{d}+f_{i}, \quad \text { when } t<t_{\text {lag }}, \\
& Y=f_{d} \exp \left(-Z_{\infty}\left(t-t_{\text {lag }}\right)\right)+f_{i}, \quad \text { when } t \geqslant t_{\text {lag }} .
\end{aligned}
$$


The $Z_{\infty}$ in this equation also is frequently written as $k$ or $k_{d}$. The conceptual difference between the three models is illustrated in Fig. 3.

\section{Model fitting}

The models were evaluated by fitting equations (5), (7), and (8) to data described above using the Marquardt method of the NLIN procedure of SAS Institute Inc. (1989) assuming constant, additive errors. Reasonable initial values are required for most non-linear regression models in order to converge. For scale estimators these are readily obtained by visual appraisal of the data. If incubation is prolonged the last observation may serve as an initial value for $\hat{f}_{i}$, whereas the observation for which $t=0$ can be used to estimate $\hat{f}_{d}+\hat{f}_{i}$. Initial values for the kinetic estimators were obtained using a grid search for $\hat{Z}_{\infty}(0.03,0.05$, and $0.07 / \mathrm{h}), \hat{t}_{\text {lag }}(2 \cdot 5$ and $5 \mathrm{~h}), \hat{Z}_{0}(0.01$ and $0.02 / \mathrm{h})$, and $\hat{k}_{z}(0 \cdot 10$ and $0 \cdot 20 / \mathrm{h})$. Alternatively, initial values for kinetic parameters may be obtained after linearization of the model.

\section{Model assessment}

Residual standard deviation (RSD) and absence of patterns in residuals served as criteria to distinguish fit between the three models. Given the data sets used, it is possible that the four-parameter models are over- or under-parameterized. The first-order model without a discrete lag time is a three-parameter model and special case of equation (5) when $Z_{0}=Z_{\infty}$, of equation (7) when $k_{z}=\infty$, and of equation (8) when $t_{l a g}=0$. The hypothesis that a firstorder model is sufficient to describe the data was tested using an $F$ test for nested models (Bates \& Watts, 1988). This test compares the residual mean square of the extra parameter added to the model with the residual mean square of the four-parameter model. Underparameterization of the models was assessed by decomposing the residual sum of squares into 'replication' sum of squares (pure error) and lack-of-fit sum of squares, and comparing the lack-of-fit mean square with the 'replication' mean square (Bates \& Watts, 1988).

\section{Parameter assessment}

It is generally not the objective of most researchers to obtain parameter estimates per se, but to determine whether the estimators change under different experimental conditions. In order to compare parameter estimates between different animals or even different studies it is desirable that parameter estimators are precise, normally distributed, unbiased, and robust (i.e. insensitive to the chosen error distribution). In contrast to linear models, least squares estimators for non-linear models are neither normally distributed nor unbiased, especially for small-to-moderate sample sizes. The extent to which properties of non-linear estimators deviate from the properties of linear estimators depends on the specific model-data set combination, as well as on the parameterization of the model. To assess behaviour of the estimators a simulation study was performed for each substrate-model combination. It was assumed that estimators from the experimental data set represented the 'real' parameters. As a result, predicted values of $Y$ represented error-free data. For each substrate-model combination, 1000 pseudo-experimental data sets were generated by adding a normally distributed, random value (with zero mean and SD 0.02) to the error-free data). Parameter estimates for each pseudo-experimental data set were obtained using the NLIN procedure of SAS Institute Inc. (1989) as previously described, using the 'real' parameters as initial values. The results of the simulation study were summarized by bias and precision. Bias was calculated as the extent to which the estimator deviated from the real parameter $(\%)$; whereas precision was calculated as the coefficient of variation of the estimator for the pseudo-experimental data.

Robustness was calculated as the change in estimator value due to a multiplicative error 


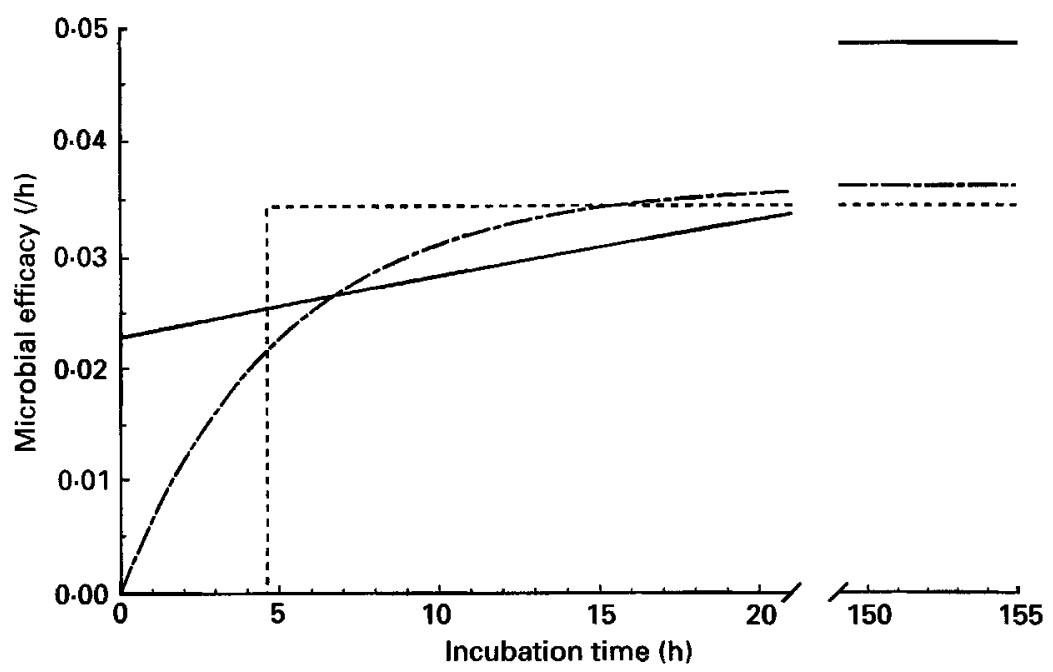

Fig. 3. Dynamics of the microbial efficacy function $(Z)$ for orchard-grass hay (Dactylis glomerata) as described by the logistic model (__ ), Gompertz-like model ( $\ldots$ - ${ }_{-}$), and discrete lag time model ( - ).

assumption compared with an additive error assumption (\%). With few replications it is generally difficult to find evidence to reject (or accept) either assumption. Estimates using the multiplicative error distribution were obtained by fitting the natural logarithms of equations (5), (7) and (8) to the natural logarithms of the data using procedures described before, e.g. for the logistic model:

$$
\ln (Y)=\ln \left(\frac{f_{d} Z_{\infty}}{Z_{0} \exp \left(Z_{\infty} t\right)+Z_{\infty}-Z_{0}}+f_{i}\right) .
$$

\section{RESULTS AND DISCUSSION}

\section{Model assessment}

With the exception of maize cobs, the residual standard deviations (RSD) were similar between models (Table 1). Moreover, the patterns of residuals were similar for the three models (see Fig. 4 as an example). The hypothesis that the rate of digestion is proportional only to the quantity of potentially digestible substrate remaining was rejected for all substrates and models $(P>0.05)$, accepting the alternative hypothesis that $Z_{0} \neq Z_{\infty}$ (equation 5), $k_{z} \neq \infty$, (equation 7), and $t_{\text {lag }} \neq 0$ (equation 8). Therefore, a first-order digestion model would be insufficient to describe the quantity of substrate remaining.

For all substrate-model combinations (except for maize cobs with the logistic and Gompertz-like models) lack-of-fit was apparent $(P<0.05)$. The lack-of-fit could not be attributed to a specific interval of incubation times. Also Robinson et al. (1986) reported that patterns of residuals were similar for several four-parameter models based on a single pool of potentially digestible material. Expansion of these models to include a second pool of potentially digestible material (i.e. the addition of two parameters) not only resulted in smaller residuals, but also increased the variation in fraction size estimates between cows (and probably lowered the precision of the estimates). Given the similarities of residuals between conceptually different models and the presence of lack-of-fit, a four-parameter 

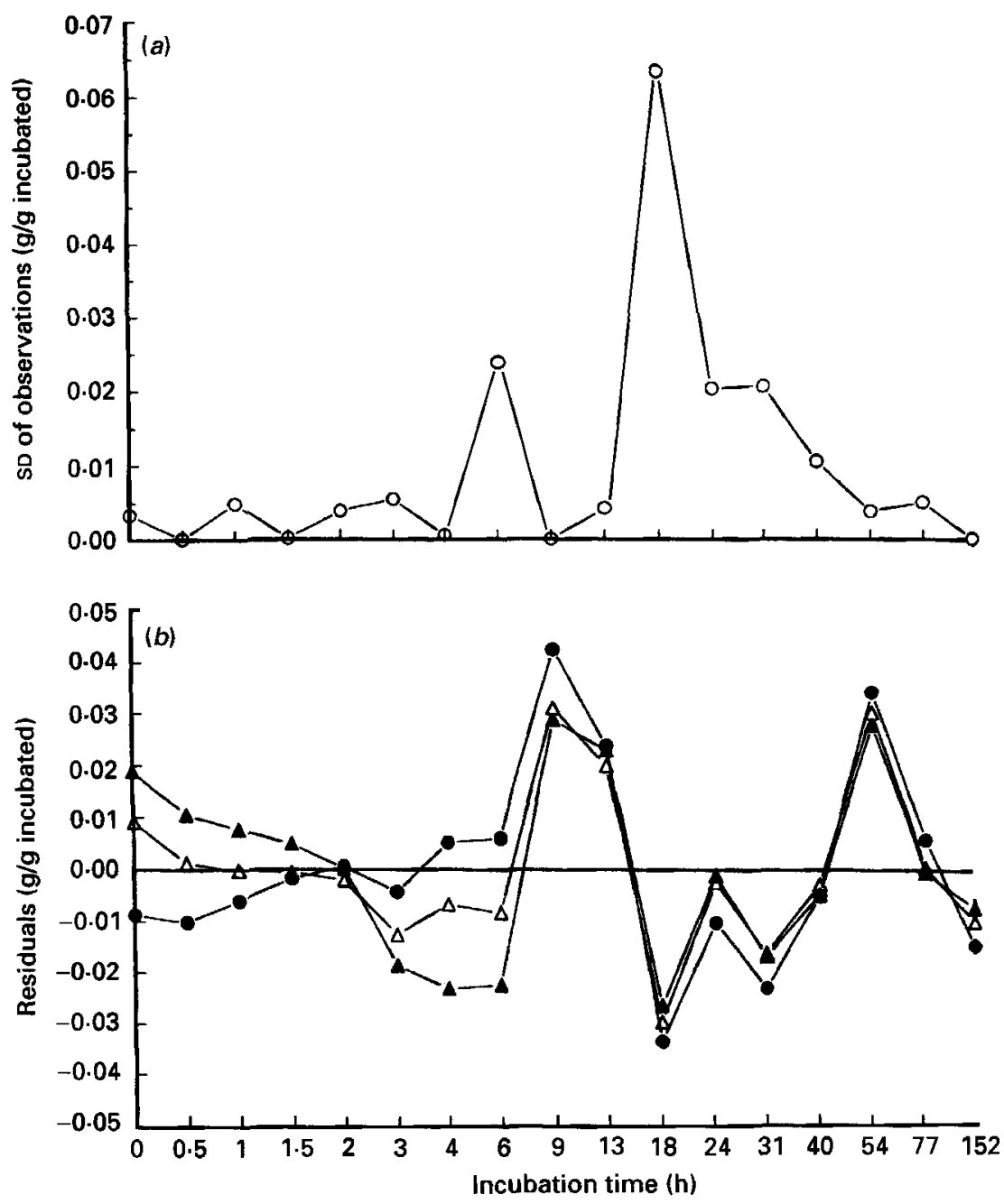

Fig. 4. (a) Standard deviation of observations $(O)$ and (b) average residual (observed value minus predicted value) as a function of incubation time for orchard-grass hay (Dactylis glomerata) as described by the logistic model ( $O)$, Gompertz-like model $(\triangle)$, and discrete lag time model $(\mathbf{A})$.

model might be insufficient to describe the data accurately. Five-parameter models could be a compromise between reducing the lack-of-fit and maintaining precision of estimators.

\section{Parameter assessment}

There were minor differences between models for estimates of the scale parameters $f_{a}$ and $f_{i}$ (Table 1). The three models mainly differed in the way the change in microbial efficacy was described (Fig. 3). The Gompertz-like and discrete lag time models assumed that the initial microbial efficacy was equal to zero. In addition, the asymptotic microbial efficacy $\left(Z_{\infty}\right)$ was of similar magnitude for these models. In contrast, the logistic model assumed that digestion started immediately upon incubation, whereas $Z_{\infty}$ was between 40 and $90 \%$ higher than for the other two models. All three models eventually evolve into a first-order model when $Z$ approaches a constant value $\left(Z_{\infty}\right)$. The discrete lag time model reaches this 
Table 1. Parameter estimates and residual standard deviations for ruminal digestion models

(Estimates with their asymptotic standard errors)

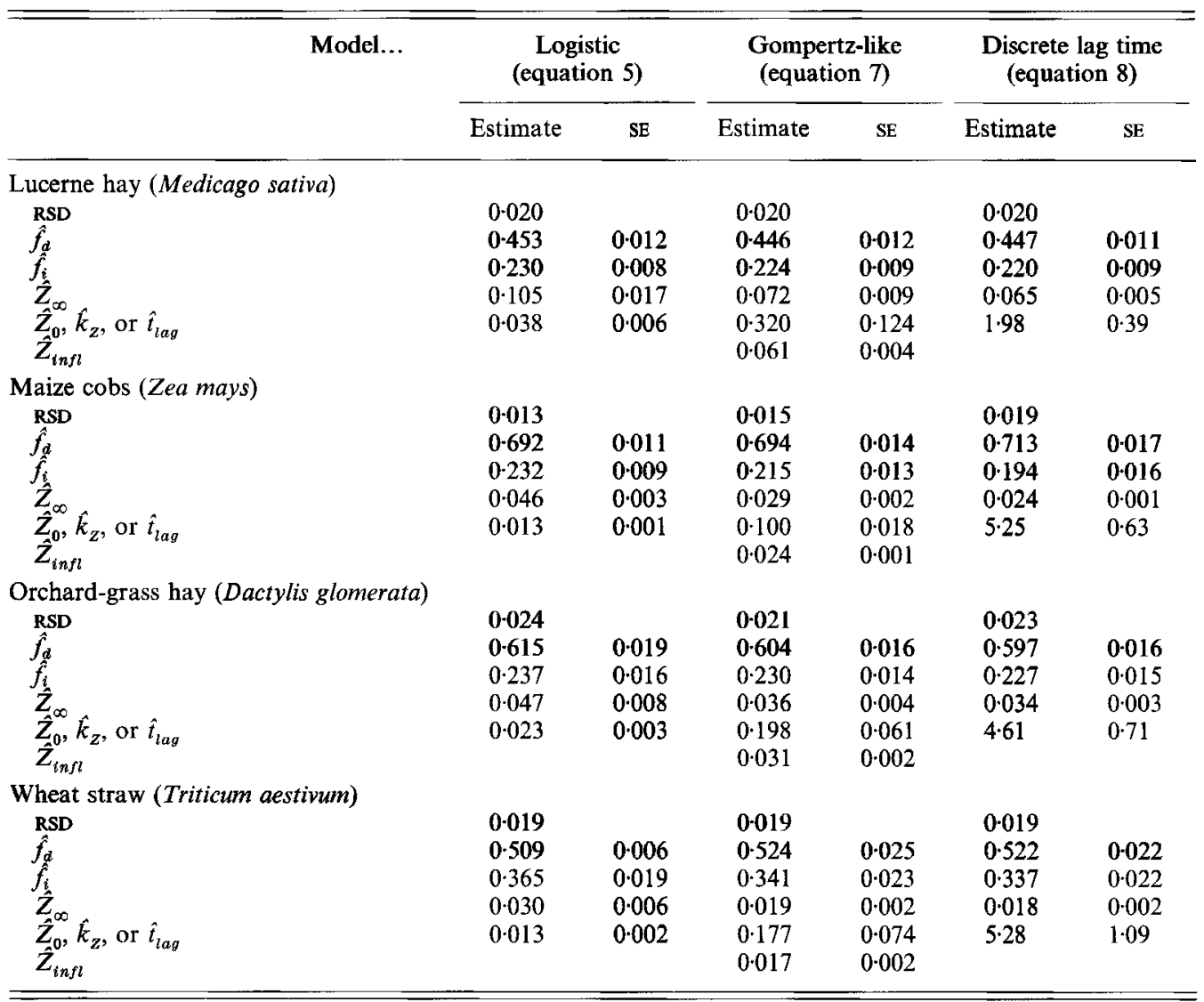

RSD, residual standard deviation ( $\mathrm{g} / \mathrm{g}$ incubated); $\hat{f}_{d}$, estimator of potentially digestible fraction $(\mathrm{g} / \mathrm{g}$ incubated); $\hat{f}_{i}$, estimator of indigestible fraction $\left(\mathrm{g} / \mathrm{g}\right.$ incubated); $\hat{Z}_{\infty}$, estimator of asymptotic microbial efficacy $(1 / \mathrm{h}) ; \hat{Z}_{0}$, estimator of initial microbial efficacy $(1 / \mathrm{h} ;$ logistic model $) ; \hat{k}_{z}$, estimator of rate of change of microbial efficacy $\left(1 / \mathrm{h}\right.$; Gompertz-like model); $\hat{t}_{\text {lag }}$, estimator of discrete lag time (h; discrete lag time model); $\hat{Z}_{\text {infl }}$, estimator of microbial efficacy at the point of inflection ( $1 / \mathrm{h}$; Gompertz-like model).

value instantaneously at $t_{l a g}$. The Gompertz-like model approached a constant value much earlier than did the logistic model. For orchard-grass hay the times required for $Z$ to reach $95 \%$ of $Z_{\infty}$ were 11.6 and $48.1 \mathrm{~h}$ for the Gompertz-like and logistic models respectively (Fig. 3).

The results of the simulation study are given in Table 2 . For all models and substrates the scale estimators $\hat{f}_{d}$ and $\hat{f}_{i}$ could be estimated with greater precision and were less biased than the other estimators $\left(\hat{Z}_{\infty}, \hat{Z}_{0}, \hat{k}_{z}\right.$, and $\left.\hat{t}_{\text {lag }}\right)$. The $\hat{k}_{z}$ was severely biased, especially for lucerne hay and wheat straw. This bias could be reduced and precision increased by replacing $\hat{k}_{z}$ with the value of $Z$ at the point of inflection of $Y\left(\hat{Z}_{i n f l}\right)$ :

$$
\hat{k}_{z}=\frac{\left(\hat{Z}_{\text {infl }}\right)^{2}}{\hat{Z}_{\infty}-\hat{Z}_{\text {infl }}} .
$$



Table 2. Coefficient of variation (averaged across substrates) and relative bias of
estimators

\begin{tabular}{|c|c|c|c|c|c|}
\hline \multirow[b]{2}{*}{ Model } & \multirow[b]{2}{*}{$\begin{array}{l}\text { Coefficient of } \\
\text { variation }(\%)\end{array}$} & \multicolumn{4}{|c|}{ Relative bias of estimators (\%) } \\
\hline & & $\begin{array}{c}\text { Lucerne hay } \\
\text { (Medicago sativa) }\end{array}$ & $\begin{array}{l}\text { Maize cobs } \\
\text { (Zea mays) }\end{array}$ & $\begin{array}{l}\text { Orchard-grass hay } \\
\text { (Dactylis glomerata) }\end{array}$ & $\begin{array}{c}\text { Wheat straw } \\
\text { (Triticum aestivum) }\end{array}$ \\
\hline \multicolumn{6}{|l|}{$\hat{f}_{d}$} \\
\hline Logistic & $3 \cdot 1$ & $0-01$ & -0.04 & -0.01 & $0-44$ \\
\hline Gompertz-like & $3 \cdot 3$ & 0.04 & -0.06 & -0.05 & -0.01 \\
\hline Discrete lag time & $3 \cdot 0$ & $0 \cdot 15$ & -0.04 & -0.03 & $0 \cdot 14$ \\
\hline \multicolumn{6}{|l|}{$\hat{f}$} \\
\hline Logistic & $5 \cdot 4$ & -0.07 & 0.04 & -0.06 & -0.65 \\
\hline Gompertz-like & $6 \cdot 2$ & 0.08 & $0 \cdot 16$ & 0.17 & $0 \cdot 10$ \\
\hline Discrete lag time & $6 \cdot 3$ & 0.05 & $0 \cdot 10$ & 0.05 & -0.05 \\
\hline \multicolumn{6}{|l|}{$\hat{Z}_{\infty}$} \\
\hline Logistic & 14.9 & 0.94 & 0.69 & 0.86 & 0.76 \\
\hline Gompertz-like & $12 \cdot 4$ & 2.52 & 2.07 & 1.56 & $2 \cdot 88$ \\
\hline Discrete lag time & $7 \cdot 4$ & 0.52 & 0.39 & 0.38 & 0.88 \\
\hline \multicolumn{6}{|l|}{$\hat{Z}_{0}, \hat{k}_{z}$ or $\hat{t}_{l a g}$} \\
\hline Logistic & 11.9 & $0 \cdot 19$ & -0.20 & -0.19 & -0.59 \\
\hline Gompertz-like & $1359 \cdot 3$ & $7 \cdot 37$ & $2 \cdot 37$ & $6 \cdot 81$ & $2 \cdot 78$ \\
\hline Discrete lag time & $18 \cdot 4$ & -1.07 & $0 \cdot 15$ & 0.01 & 0.29 \\
\hline \multicolumn{6}{|l|}{$\hat{Z}_{\text {infl }}$} \\
\hline Gompertz-like & $7 \cdot 0$ & 0.77 & 0.58 & 0.58 & $1 \cdot 34$ \\
\hline
\end{tabular}

$\hat{f}_{d}$, estimator of potentially digestible fraction $(\mathrm{g} / \mathrm{g}$ incubated $) ; \hat{f}_{i}$, estimator of indigestible fraction $(\mathrm{g} / \mathrm{g}$ incubated); $\hat{Z}_{\mathrm{c}}$, estimator of asymptotic microbial efficacy $(1 / \mathrm{h}) ; \hat{Z}_{0}$, estimator of initial microbial efficacy $(1 / \mathrm{h}$; logistic models); $\hat{k}_{z}$, estimator of rate of change of microbial efficacy $\left(1 / \mathrm{h}\right.$; Gompertz-like model); $\hat{i}_{\text {lag }}$, estimator of discrete lag time $(\mathrm{h} ;$ discrete lag time model $) ; Z_{i n f l}$, estimator of microbial efficacy at the point of inflection $(1 / \mathrm{h}$; Gompertz-like model).

Estimators of the Gompertz-like model (when parameterized to include $\hat{Z}_{\text {infl }}$ ) and logistic model were more robust than those of the discrete lag time model (Table 3). The $\hat{t}_{\text {lag }}$ was neither a robust nor a precise estimator. Similar results were found by Fadel $(1992 a)$. Scale estimators appeared to be more robust than the kinetic estimators.

There were many cases in the simulation study where the discrete lag time model converged with $\hat{t}_{\text {lag }}$ equal to a value of the independent variable (incubation time). If rounded to three decimal places and assuming a normal distribution for $\hat{t}_{\text {lag }}$, the theoretical probability for this to occur would range from 0.0004 for maize cobs to 0.0016 for lucerne hay. However, the simulation study indicated that this probability ranged from 0.048 for maize cobs to 0.187 for lucerne hay. The estimator $\hat{t}_{\text {lag }}$ is therefore strongly biased towards obtaining estimates equal to a value of the independent variable. This is caused by the fact that the first-derivative of equation (8) with respect to $t_{l a g}$ is not defined, and many algorithms (like the ones used by SAS Institute Inc., 1989) require this derivative. The firstderivative is defined for each of the two segments of equation (8) (which were the ones defined for the Marquardt method), but not for the whole model.

Although the experimental data indicated that $Z$ varied with incubation time, this was not apparent for all pseudo-experimental data. In the logistic model, $Z$ will vary with incubation time only when $Z_{0}+Z_{\infty}$. In twenty-two of the 4000 pseudo-experimental data sets (1000 for each substrate) the $\hat{Z}_{0}: \hat{Z}_{\infty}$ ratio exceeded 0.9 and in seventeen cases it even exceeded $1 \cdot 0$. The $\hat{k}_{z}$ exceeded $1.0 / \mathrm{h}$ in thirty-four of the 4000 pseudo-experimental data 
Table 3. Parameter estimators assuming a multiplicative error distribution

(Estimators, with changes in percentage relative to assuming additive error distribution in parentheses)

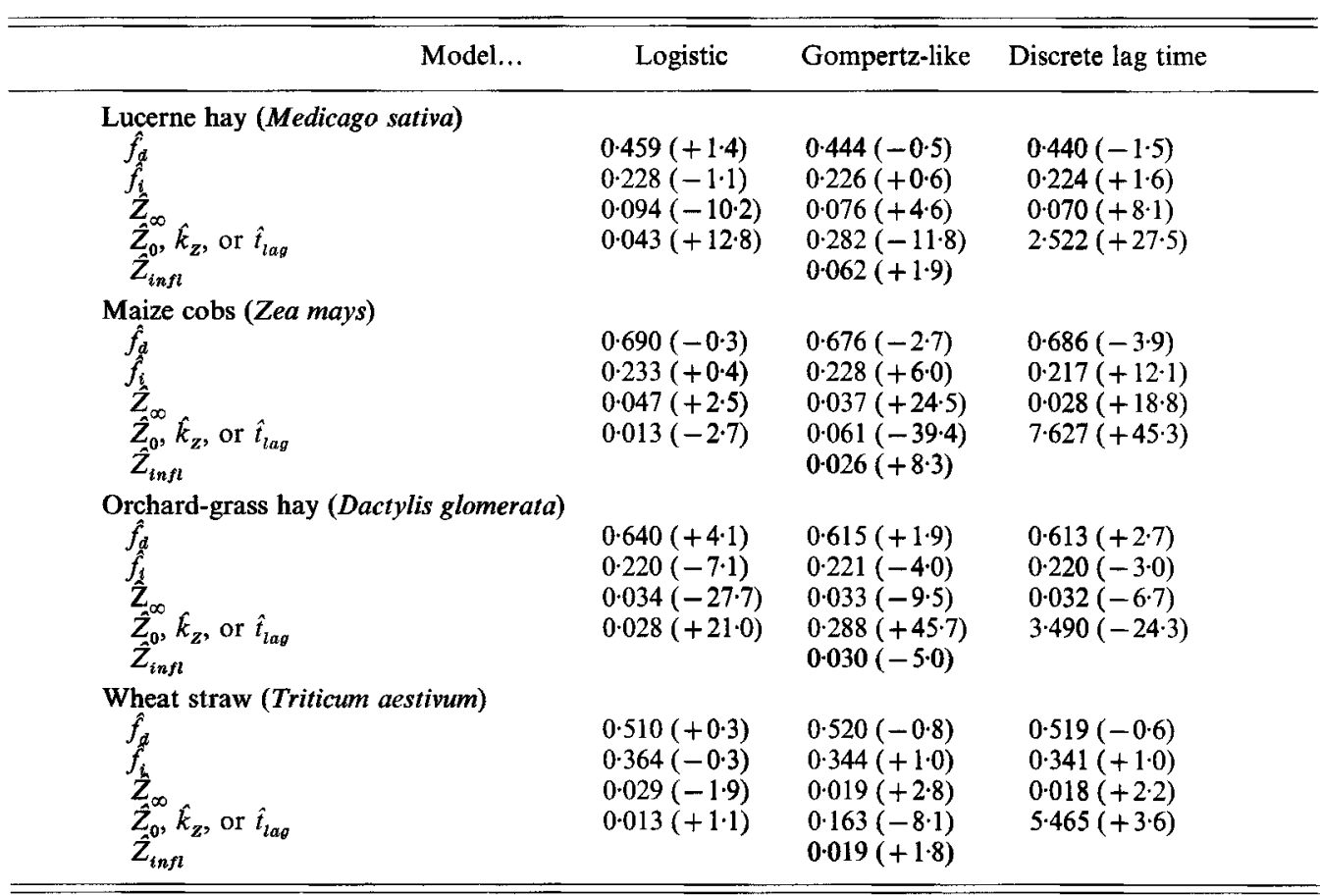

$\hat{f}_{d}$, estimator of potentially digestible fraction $(\mathrm{g} / \mathrm{g}$ incubated $) ; \hat{f}_{\hat{l}}$, estimator of indigestible fraction $(\mathrm{g} / \mathrm{g}$ incubated); $\hat{Z}_{\infty}$, estimator of asymptotic microbial efficacy $(1 / \mathrm{h}) ; \hat{Z}_{0}$, estimator of initial microbial efficacy $(1 / \mathrm{h}$; logistic model); $\hat{k}_{z}$, estimator of rate of change of microbial efficacy $(1 / \mathrm{h} ;$ Gompertz-like model $) ; \hat{t}_{l a g}$, estimator of discrete lag time (h; discrete lag time model); $\hat{Z}_{i n f}$, estimator of microbial efficacy at the point of inflection $(1 / \mathrm{h}$; Gompertz-like model).

sets. This is also the main cause of the bias and high coefficient of variation reported in Table 2.

\section{Mathematical and biological properties}

The discrete lag time model has been parameterized to describe the quantity of substrate remaining (e.g. Mertens \& Loften, 1980) or the quantity of substrate digested (e.g. McDonald, 1981) even though they are based on the same conceptual model. Rate of digestion is defined as the first-derivative of the equation with respect to time $(\mathrm{d} Y / \mathrm{d} t=$ $\mathrm{d} S / \mathrm{d} t)$. It should be noted that this differs from the fractional rate of digestion $((\mathrm{d} s / \mathrm{d} t) / S)$. The rate of digestion would be negative and increasing (after $t_{\text {lag }}$ ) if the equation is parameterized to describe the quantity of substrate remaining $(Y)$, but positive and decreasing when parameterized to describe the quantity of substrate digested $(1-Y)$, even though the absolute values of the first-derivatives are equal. To avoid confusion, the rate of digestion is defined here as the absolute value of the first-derivative of the equation.

In first-order kinetics, rate of digestion is a continuous declining function where each subsequent unit of potentially digestible substrate is more difficult to digest. This may be caused by build-up of lignin-carbohydrate complexes (or lack of available potentially digestible substrate) on the surface during the digestion process (Chesson, 1993). However, 
it is likely that initially the microbial efficacy is more limiting than the absence of potentially digestible substrate. Because the microbial efficacy will increase through autocatalysis and entry of microbes from the environment, the rate of digestion will also increase. Eventually, as incubation time proceeds, the negative effects of lack of available potentially digestible substrate on the surface will outweigh the beneficial effects of additional microbes, and the rate of digestion will decline. At the point of inflection of $Y$, the acceleration of digestion (second derivatives of equations (5) and (7)) equals zero and the fractional change in microbial efficacy equals the fractional rate of digestion (i.e. $(\mathrm{d} Z / \mathrm{d} t) / Z=|(\mathrm{d} S / \mathrm{d} t) / S|=$ $\left.Z_{\text {infl }}\right)$. In biological terms, the beneficial effect of additional microbes equals the negative effect caused by lack of available potentially digestible substrate on the surface. Therefore, the point of inflection indicates the transition from the microbe-limiting phase to the substrate-limiting phase. The Gompertz-like model (equation 7) always has a point of inflection, whereas the logistic model (equation 5) has a point of inflection only when $\left(Z_{\infty}-Z_{0}\right)>Z_{0}$. In contrast, the discrete lag time model (equation 8) assumes an abrupt, rather than a smooth, transition between the two phases of digestion.

The logistic equation was derived by assuming there is no exchange of microbes between the system and its environment (Fig. 2) and it is the only model based solely on autocatalysis. This implies that all produced microbes will participate in digestion of substrate they originated from. As a result, the rate of change of microbial efficacy $(\mathrm{d} Z / \mathrm{d} t)$ is proportional to the rate of digestion $(\mathrm{d} S / \mathrm{d} t)$ and the point of inflection is the centre of symmetry. The extent to which autocatalysis contributes to digestion might be due to physical characteristics of the particle. The outer surfaces of plant particles are protected by waxes and the cuticle which appear to be inert to ruminal microbes (Chesson \& Forsberg, 1988). Therefore, digestion has to occur from within and the site of digestion (i.e. the inner particle) is most probably also the site of synthesis of microbial mass. If sufficient potentially digestible substrate is available, these newly produced microbes will attach to sites within the same particle. This is more likely to happen when the inner particle is protected from the environment (e.g. when particles are large and intact). However, the initial population of microbes within a particle has to originate from the ruminal fluid (actually, they detach from other particles).

The inclusion of a discrete lag time finds its origin in the inadequacy of a first-order model to describe digestion during the early hours of incubation. During the lag phase digestion does not occur due to limited hydration and microbial colonization of substrate. During the digestion phase (i.e. when $t>t_{l a g}$ ) digestion is limited only by the quantity of potentially digestible substrate remaining. As a result it is implied that substrate is hydrated and colonized instantaneously at $t_{\text {lag }}$. Although all-or-none processes may exist in biology, this seems an unlikely scenario for hydration and microbial colonization.

In situ and in vitro incubation procedures only account for disappearance of substrate through digestion but ignore passage to the lower gastrointestinal tract. Ørskov \& McDonald (1979) proposed a method for calculating the effective degradation $(P)$ accounting for both digestion and passage. If $k_{p}$ is the fractional rate of passage $(/ \mathrm{h})$ and $S^{\prime}$ the quantity of potentially digestible substrate remaining subjected to both passage and digestion, the rate of disappearance is given by:

$$
\frac{\mathrm{d} S^{\prime}}{\mathrm{d} t}=\left(-k_{p}+Z\right) S^{\prime}, \quad \text { with } S^{\prime}(0)=f_{d}
$$

The effective degradability is then given by:

$$
P=f_{s}+\int_{0}^{\infty}\left(Z S^{\prime}\right) \mathrm{d} t
$$


Analytical solutions exist for $S^{\prime}$ for all three models, but for $P$ only for the model with a discrete lag time. However, solutions can easily be obtained through numerical integration.

\section{Similar models}

Several other workers have used logistic models to describe in situ digestion. Wadsworth et al. (1983) used a three-parameter logistic function to describe the change in the fractional rate of digestion. To describe in situ digestion, integration of such an equation would result in a five-parameter model. Robinson et al. (1986) derived the four-parameter logistic model from hypotheses similar to those described above:

$$
Y=\frac{f_{d}}{p \exp (w t)+(1-p)}+f_{i}
$$

It can be shown that this equation is a different parameterization of equation (5), where $w=Z_{\infty}$ and $p=Z_{0} / Z_{\infty}$. As for certain cases in the simulation study, Robinson et al. (1986) reported that $p$ exceeded 1.0 for some substrates. Even though such a model is valid mathematically, its biological interpretation would imply that the microbial efficacy declines rather than increases with time. France et al. (1990) derived the logistic function from the hypothesis that specific microbial growth rate is proportional to the quantity of potentially digestible substrate remaining.

As in the present study, Mertens (1973) suggested that the fractional rate of digestion increased with incubation time (from zero to a maximum $Z_{\infty}$ ), and proposed the following equation:

$$
Y=f_{d} \exp \left(-Z_{\infty}\left(1-\exp \left(-k_{z} t\right)\right) t\right)+f_{i} .
$$

However, in this equation the term $Z_{\infty}\left(1-\exp \left(-k_{z} t\right)\right)$ does not represent the fractional rate of digestion. It can be shown that $Z_{\infty}$ is the asymptote, but that the maximum fractional rate of digestion in this equation is $Z_{\infty}(1+\exp (-2))$, which occurs at $t=2 / k_{z}$. The reason for the difference between Mertens' equation and equation (7) is that Mertens (1973) included the term $Z_{\infty}\left(1-\exp \left(-k_{z} t\right)\right)$ directly in the first-order model, whereas in the present study it was included in the differential equation (after which it was integrated with respect to time).

Sauvant et al. (1985) used a three-parameter Gompertz model to describe digestion of substrate. Although such a model might fit the data well, there is an important difference between the Gompertz model and the Gompertz-like model derived above. The rate of digestion in the Gompertz model is the product of the quantity of digested substrate $(1-Y$; which is continuously increasing) and a function that declines autonomously with time. In contrast, the rate of digestion for equation (7) equals the product of the quantity of potentially digestible substrate remaining ( $Y$; which is continuously declining) and function that increases autonomously with time. France et al. (1990) derived a Gompertz function from the hypothesis that microbial mass can be described by an exponentially increasing function. As a result the microbial efficacy function $Z$ does not approach an asymptote, but increases exponentially. Another parameterization of the Gompertz function was found when existence of an inhibition function for digestion was assumed (e.g. through build-up of lignified material; France et al. 1990). Under this assumption the function $Z$ approaches zero for infinite incubation time. An advantage of the Gompertz-like model published in the present study is that three of the four parameters $\left(f_{d}, f_{i}\right.$, and $\left.Z_{\infty}\right)$ can be interpreted as in the model with the discrete lag time; only $t_{\text {lag }}$ is replaced by either $k_{Z}$ or $Z_{\text {infl }}$.

Van Milgen et al. (1991) proposed a sequential, two-compartment model to describe in situ digestion, assuming that not all potentially digestible substrate was available for digestion upon incubation. Once it became available it was digested according to a first- 
order process (constant microbial efficacy). In contrast, the sigmoidal models presented here assume that all potentially digestible substrate is available upon incubation, but that digestion is determined by changing microbial efficacy.

Because digestion is a surface phenomenon, rate of digestion is determined by the spatial distribution of potentially digestible substrate (i.e. embedded in tissues or present on the surface), availability of microbes on the surface, and the total surface area. Although models can be developed that include elements of these concepts, these models would require additional parameters to be estimated. The presence of lack-of-fit for fourparameter models might justify such an approach.

Compared with other studies using the in situ technique the present study was performed with a rather large number (17) of incubation time points. Most in situ studies are performed with six to ten time points with the objective to compare digestion kinetics of different feedstuffs, or of the same feedstuff under different conditions. In these studies a model is selected as the 'true' model and the researcher is interested in the effect of a treatment on parameter estimates. In contrast, the objective of the present study was to compare different, competing models. The partitioning of a limited number of bags between different incubation time points and replicates depends largely on the validity of the model. If a model is selected as the 'true' model, studies with few data points are possible. Theoretically, a D-optimal design (Box \& Lucas, 1959; four time points for the present models, using additional bags for replicates) would be the most favourable design. This design minimizes the determinant of the variance-covariance resulting in accurate parameter estimates and minimal correlation between estimators. However, Fadel (1992 b) showed that these designs are not very robust and accurate information about the parameters values would be required a priori to calculate design points. In contrast, if the validity of a model is being questioned, each bag should be used for a different incubation time. Intermediate designs are possible if both objectives (accurate estimates and uncertainty about the model) are important.

\section{CONCLUSION}

The purpose of fitting a model to the data is to reduce the amount of information provided by the observations, preferably to a small number of biologically interpretable estimators. Criteria to assess how well this is done include error behaviour, properties of the estimators and, above all, the biological reasoning for choosing a certain model. Presence of lack-offit and the similarity of patterns of residuals suggest that a four-parameter model is insufficient to describe the data accurately. If a four-parameter model is to be used, no model was clearly better than the others (based on statistical criteria). The biased estimator $\hat{k}_{z}$ in the Gompertz-like model could easily be replaced by the robust estimator $\hat{Z}_{i n f t}$. Although widely used, the biological basis for the discrete lag time model is rather limited. It supposes that limiting factors to digestion (e.g. hydration and microbial colonization) are overcome instantaneously at lag time. Moreover, the $\hat{t}_{\text {lag }}$ cannot be estimated with great precision, is sensitive to the error assumption, and (depending on the statistical package used) may be strongly biased towards obtaining estimates equal to a value of the independent variable. Both the logistic and the Gompertz-like model are suitable alternatives for the discrete lag time model. Although the logistic model specifically accounts for autocatalysis, it does not account for microbial migration between the ruminal fluid and the substrate particle (or nylon bags). As a result it has some biologically inappropriate properties (like a non-zero initial value for $Z$ ). The Gompertz-like model neither specifically describes autocatalysis nor microbial migration but uses a more generalized, time-dependent approach in describing the change in the microbial efficacy. 
The choice between either model depends on how the incorporation of autocatalysis (but lack of microbial migration) in the logistic model is perceived compared with a more generalized approach in the Gompertz-like model.

\section{REFERENCES}

Bates, D. M. \& Watts, D. G. (1988). Nonlinear Regression Analysis and its Applications. New York: John Wiley \& Sons.

Box, G. E. P. \& Lucas, H. L. (1959). Design of experiments in non-linear situations, Biometrika 46, $77-90$.

Cheng, K.-J., Fay, J. P., Howarth, R. E. \& Costerton, J. W. (1980). Sequence of events in the digestion of fresh legume leaves by rumen bacteria. Applied and Environmental Microbiology 40, 613-625.

Chesson, A. (1993). Mechanistic models of forage cell wall degradation. In Forage Cell Wall Structure and Digestibility, pp. 347-376 [H. G. Jung, D. R. Buxton, R. D. Hatfield and J. Ralph, editors]. Madison, WI: American Society of Agronomy, Crop Science Society of America, and Soil Science Society of America.

Chesson, A. \& Forsberg, C. W. (1988). Polysaccharide degradation by rumen microorganisms. In The Rumen Microbial Ecosystem, pp. 251-284 [P. N. Hobson, editor]. London: Elsevier Applied Science.

Fadel, J. G. (1992a). Application of theoretically optimal sampling schedule designs for fiber digestion estimation in sacco. Journal of Dairy Science 75, 2184-2189.

Fadel, J. G. (1992b). Simulation of traditional and optimal sampling schedule designs for digestion and passage models. Journal of Theoretical Biology 157, 109-126.

France, J., Thornley, J. H. M., Lopez, S., Siddons, R. C., Dhanoa, M. S., Van Soest, P. J. \& Gill, M. (1990). On the two-compartment model for estimating the rate and extent of feed degradation in the rumen. Journal of Theoretical Biology 146, 269-287.

McDonald, I. (1981). A revised model for the estimation of protein degradability in the rumen. Journal of Agricultural Science, Cambridge 96, 251-252.

Mertens, D. R. (1973). Application of theoretical mathematical models to cell wall digestion and forage intake in ruminants. PhD Thesis, Cornell University, Ithaca, NY, USA.

Mertens, D. R. \& Loften, J. R. (1980). The effect of starch on forage fiber digestion kinetics in vitro. Journal of Dairy Science 63, 1437-1446.

Grskov, E. R. \& McDonald, I. (1979). The estimation of protein degradability in the rumen from incubation measurements weighted according to rate of passage. Journal of Agricultural Science, Cambridge $92,499-503$.

Robinson, P. H., Fadel, J. G. \& Tamminga, S. (1986). Evaluation of mathematical models to describe neutral detergent residue in terms of its susceptibility to degradation in the rumen. Animal Feed Science and Technology $15,249-271$.

SAS Institute Inc. (1989). SAS/STAT User's Guide, Version 6, Volume 2, 4th ed. Cary, NC: SAS Institute Inc.

Sauvant, D., Bertrand, D. \& Giger, S. (1985). Variations and prevision of the in sacco dry matter digestion of concentrates and by-products. Animal Feed Science and Technology 13, 7-23.

van Milgen, J. (1994). Autocatalytic models describing ruminal in situ digestion. Annales de Zootechnie 43, 275.

van Milgen, J., Berger, L. L. \& Murphy, M. R. (1993). An integrated, dynamic model of feed hydration and digestion, and subsequent bacterial mass accumulation in the rumen. British Journal of Nutrition 70, $471-483$.

van Milgen, J., Murphy, M. R. \& Berger, L. L. (1991). A compartmental model to analyse ruminal digestion. Journal of Dairy Science 74, 2515-2529.

Wadsworth, J. C., Sriskandarajah, N. \& Kellaway, R. C. (1983). Mathematical description of delays in onset of ruminal fibre digestion. In Fibre in Human and Animal Nutrition. Proceedings of Dietary Fibre in Human and Animal Nutrition Symposium, p. 112 [G. Wallace and L. Bell, editors]. Wellington, New Zealand: The Royal Society of New Zealand. 